\title{
Aortic Pulse Wave Velocities Using Real-Time Phase-Contrast MRI
}

\author{
Arun A. Joseph ${ }^{\mathrm{a}, \mathrm{b}}$, Martin Fasshauser ${ }^{\mathrm{c}}$, Klaus-Dietmar Merboldt ${ }^{\mathrm{a}}$ and Jens Frahm ${ }^{\mathrm{a}, \mathrm{b}, *}$ \\ ${ }^{a}$ Biomedizinische NMR Forschungs GmbH am Max-Planck-Institut für biophysikalische Chemie, Göttingen, Germany \\ ${ }^{b}$ DZHK, German Center for Cardiovascular Research, partner site Göttingen, Germany \\ ${ }^{c}$ Abteilung Diagnostische Radiologie, Universitätsmedizin, Göttingen, Germany
}

\begin{abstract}
Purpose: To evaluate aortic pulse wave velocities obtained by real-time phase-contrast (PC) MRI in comparison to cine PC MRI.

Methods: Real-time PC MRI of eight healthy volunteers employed highly undersampled radial FLASH sequences and phase-sensitive image reconstructions by regularized nonlinear inversion (NLINV) at $40 \mathrm{~ms}$ temporal resolution and 1.3 $\mathrm{mm}$ in-plane resolution. Pulse wave velocities were analyzed for combinations of 2, 3 and 4 locations of aortic flow using time-to-upslope and cross-correlation methods.

Results: For the time-to-upslope analysis mean pulse wave velocities ranged from 3.5 to $3.9 \mathrm{~m} \mathrm{~s}^{-1}$ for real-time PC MRI and from 3.5 to $3.8 \mathrm{~m} \mathrm{~s}^{-1}$ for cine PC MRI. A cross-correlation analysis of the same data resulted in 2.9 to $3.3 \mathrm{~m} \mathrm{~s}^{-1}$ and 3.3 to $3.7 \mathrm{~m} \mathrm{~s}^{-1}$, respectively.

Conclusion: Real-time PC MRI determined aortic pulse wave velocities from single cardiac cycles in close correspondence to values obtained by cine PC MRI.
\end{abstract}

Keywords: Real-time MRI, Phase-contrast MRI, Pulse wave velocity, Blood flow.

\section{INTRODUCTION}

The pulse wave velocity (PWV) describes the pulse wave of blood flow through the aorta and reflects its elastic properties $[1,2]$. It is considered as a biomarker or indicator of aortic stiffness [3] which is an important sequela of diseases such as artherosclerosis [4], stroke [5], and hypertension [6]. PWV is usually calculated from the distance travelled by the pulse wave between at least two locations along the aorta divided by the time needed. This transit time may be obtained from respective flow curves using different proposed analysis methods (see below). A pressure catheter is considered as gold standard for aortic PWV determination, but is not frequently applied due to its invasive nature [7]. Alternative methods determine peripheral PWV by a variety of tools such as, for example, sphygmomanometers in order to derive aortic PWV with the use of invasively validated algorithms [8-10]. Instead of these approaches, however, the focus has shifted to the use of cine phase-contrast (PC) MRI where the acquisition extends over multiple heartbeats with synchronization to the electrocardiogram (ECG), but allows for noninvasive flow velocity measurements from arbitrary positions along the aorta [11-13]. The method determines the velocity of through-plane flow in a single averaged cardiac cycle

*Address correspondence to this author at the Biomedizinische NMR Forschungs GmbH am Max-Planck-Institut für biophysikalische Chemie, 37070 Göttingen, Germany; E-Mail: jfrahm@gwdg.de which is retrospectively reconstructed from multiple heartbeats. It may be extended to multi-dimensional PC MRI which encodes velocity in three dimensions at the expense of prolonged measuring time $[14,15]$. In fact, cine 2D PC MRI and 4D PC MRI methods under free breathing conditions have typical measuring times of about $3.5 \mathrm{~min}$ and at least 10 min, respectively. Therefore, real-time MRI techniques using echo-planar [16], spiral [17-20] or radial encoding strategies $[21,22]$ in combination with data undersampling and parallel imaging [23-25] gained increasing interest for flow assessments. However, only few studies using one-dimensional MRI techniques have attempted to analyze PWV in real time $[26,27]$. This situation may now be overcome by recent advances in real-time MRI which combine highly undersampled radial fast low-angle shot (FLASH) acquisitions with image reconstructions by regularized nonlinear inversion (NLINV) [28-32]. This technique has been adapted to PC MRI to obtain flow information in real time [33-36]. The aim of this study was to evaluate the performance and potential of PWV determinations based on real-time PC MRI in comparison to conventional cine PC MRI.

\section{METHODS}

\section{Subjects}

Nine young volunteers ( 7 male, 2 female, mean age 26.8 \pm 3.3 years) without known cardiovascular illness were 


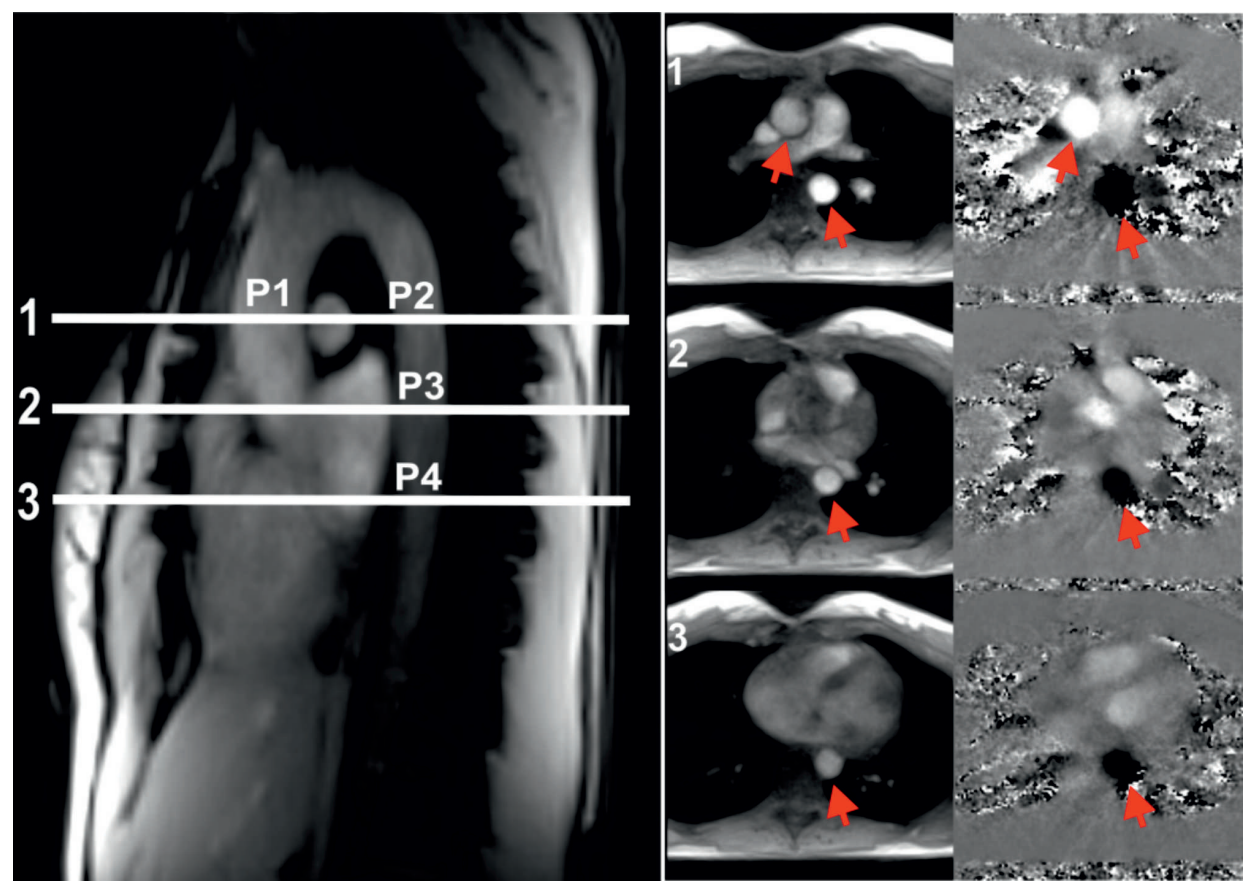

Fig. (1). MRI of aortic blood flow. (Left) Sections 1, 2 and 3 selected for measurements in positions P1, P2, P3 and P4. (Right) Corresponding magnitude images and phase-difference maps obtained from real-time phase-contrast MRI (arrows $=$ vascular lumen of interest).

recruited for this study. One male subject had to be excluded because of abnormal high pulse wave velocities. Written informed consent, according to the recommendations of the local ethics committee, was obtained from all subjects prior to MRI.

\section{MRI}

All experiments were performed on a 3 Tesla (T) MRI system (Magnetom TIM Trio, Siemens Healthcare, Erlangen, Germany) with the use of a 16-channel anterior and 16channel posterior body receive coil. Flow velocities in the aorta were obtained using real-time and electrocardiogram(ECG) synchronized cine PC MRI under free breathing conditions. Real-time PC MRI was based on highly undersampled radial FLASH acquisitions with phase-sensitive NLINV reconstruction as previously described [33, 34]. The temporal accuracy of NLINV reconstructions has experimentally been confirmed [37], in particular for situations without temporal filtering as used here for real-time PC velocity maps. All PC MRI measurements were performed with an in-plane resolution of $1.3 \mathrm{~mm}$, slice thickness of $6 \mathrm{~mm}$, and velocity-encoding gradients corresponding to a maximum velocity of $200 \mathrm{~cm} \mathrm{~s}^{-1}$. Other parameters for free-breathing cine PC MRI with Cartesian encoding and retrospective sorting (standard sequence of the vendor) were: $\mathrm{TR}=20.05 \mathrm{~ms}$, $\mathrm{TE}=2.18 \mathrm{~ms}$, flip angle $=25$ degree, 3 averages, 30 cardiac phases, field of view $(\mathrm{FOV})=320 \times 320 \mathrm{~mm}^{2}$, base resolution $256 \times 256 \mathrm{~mm}^{2}$, measuring time $3.5 \mathrm{~min}$.

Real-time PC MRI with undersampled radial encoding employed the following parameters: $\mathrm{TR}=2.86 \mathrm{~ms}$, $\mathrm{TE}=$ $1.93 \mathrm{~ms}$, flip angle $=10$ degree, 7 radial spokes per image (with and without flow-encoding gradient in a sequential acquisition mode), FOV $=192 \times 192 \mathrm{~mm}^{2}$, base resolution $144 \times 144 \mathrm{~mm}^{2}$, measuring time $40 \mathrm{~ms}$ for each pair of magnitude image and phase-difference map. Typically, real-time acquisitions were performed for a period of $15 \mathrm{~s}$ corresponding to 375 magnitude images and PC maps. Online reconstruction and display of real-time images with minimal delay was achieved by a parallelized version of the NLINV algorithm [38] and a bypass computer (sysGen/TYAN OctupleGPU, Sysgen, Bremen, Germany) which was equipped with 8 graphical processing units (GeForce GTX, TITAN, NVIDIA, Santa Clara, CA) and fully integrated into the reconstruction pipeline of the commercial MRI system.

Three slice positions covering four locations along the aorta were chosen for flow measurements and PWV evaluations as shown in Fig. (1). Real-time PC MRI for the first slice was repeated three times to evaluate the reproducibility of aortic PWV determinations from just one section (or two positions). The total examination time per subject, including the long cine PC MRI acquisitions, was about $30 \mathrm{~min}$.

\section{Flow and Aortic PWV Analysis}

The quantitative flow analysis of PC MRI data was performed with prototype software (Fraunhofer MEVIS, Bremen, Germany), especially modified for the automated analysis of real-time MRI acquisitions. Flow evaluations were performed for 10 consecutive cardiac cycles. As common practice for PC MRI, PWV determinations were based on the time courses of mean velocities (i.e., spatially averaged across the aortic lumen) which were fitted with in-house programs written in Matlab (Mathworks, USA). The 


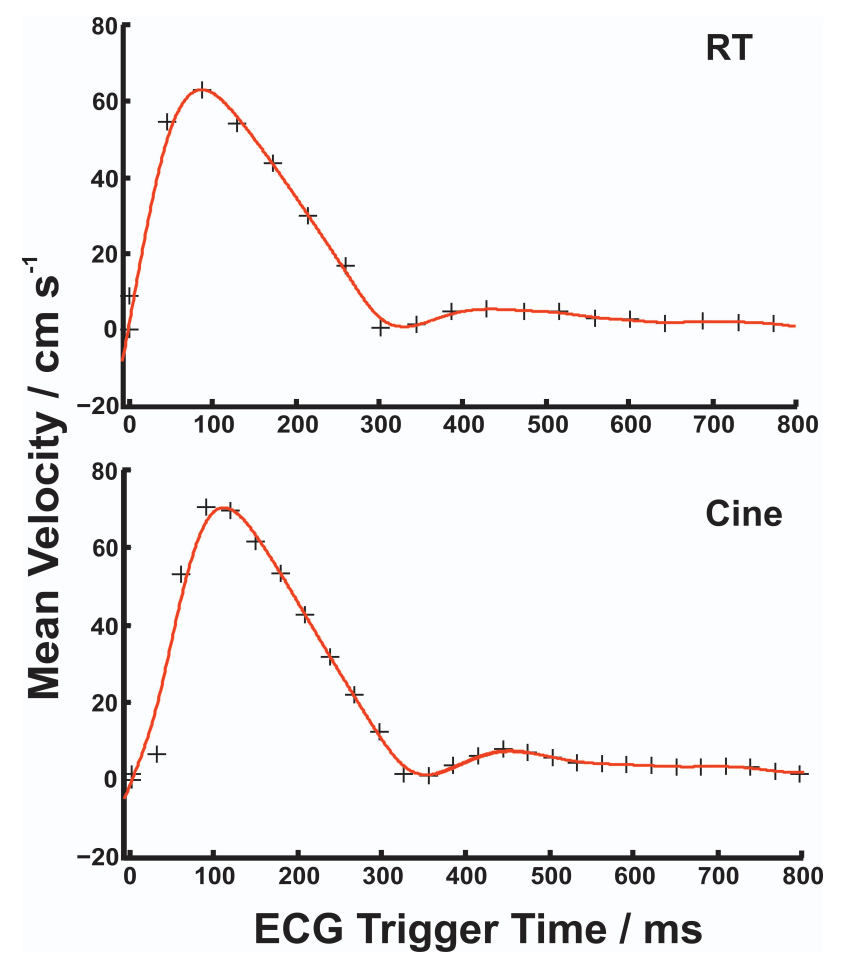

Fig. (2). Mean blood flow velocities in the ascending aorta. Realtime (RT) and cine (Cine) phase-contrast MRI data as a function of time after the $\mathrm{R}$ wave.

analyses were performed for two positions (P1, P2), three positions $(\mathrm{P} 1, \mathrm{P} 3, \mathrm{P} 4)$ and four positions $(\mathrm{P} 1, \mathrm{P} 2, \mathrm{P} 3, \mathrm{P} 4)$ as shown in Fig. (1). Respective distances were estimated by taking the center line along the aorta using Syngo MR (Siemens Healthcare, Erlangen, Germany).

The transit times between different regions were measured according to previously proposed methods characterized as time-to-foot, time-to-peak, time-to-upslope (TTU) and cross correlation (XCor), see [15, 39-42] for detailed definitions. However, because preliminary trials proved TTU and XCor to be much more robust than the first two approaches which rely on the correct identification of only a single data point, only TTU and XCor were chosen for the analyses presented here. TTU is defined as the midpoint of the velocity upslope to peak systole. It is obtained by fitting a straight line between data points within an interval given by 20 to $80 \%$ of the maximum velocity. The XCor method employs a cross-correlation of the velocity time courses for two locations and thereby directly determines the relative time shift.

\section{RESULTS}

Fig. (2) depicts the mean velocities in the ascending aorta as a function of time after the R wave for real-time PC MRI (single cycle) and cine PC MRI. The resulting absolute velocities as well as the overall time courses and respective fits are very similar in all cases. The aortic PWV values obtained for real-time PC MRI in a single section ( 2 positions) are summarized in Table $\mathbf{1}$ for three consecutive measurements per subject and both TTU and XCor analysis. The values correspond to the mean and standard deviation obtained for 10 consecutive cardiac cycles. It turns out that the XCor analysis results in systematically lower PWV values than the TTU method. In any case, in most subjects real-time PC MRI is characterized by a high intrasubject reproducibility. Nevertheless, physiological variations such as an initial excitement or arousal during the first measurement cannot be excluded as, for example, seen in subjects \#6 and \#8.

Table 2 compares corresponding mean values averaged across subjects for real-time PC MRI and cine PC MRI for both analysis methods and 2, 3 and 4 locations. In particular for the TTU method, PWV values are in close agreement for both techniques. It should be noted that the standard deviations for real-time PWV values predominantly reflect beatto-beat variations in response to true physiological influences. These effects include the aforementioned differences

Table 1. Aortic pulse wave velocities using real-time phase-contrast MRI.

\begin{tabular}{|c|c|c|c|c|c|c|}
\hline \multirow{2}{*}{ Subject } & \multicolumn{3}{|c|}{ Time To Upslope } & \multicolumn{3}{c|}{ Cross Correlation } \\
\cline { 2 - 6 } & $\mathbf{1}$ & $\mathbf{2}$ & $\mathbf{3}$ & $\mathbf{1}$ & $\mathbf{2}$ \\
\hline \hline$\# 1$ & $4.0 \pm 1.2$ & $4.3 \pm 1.1$ & $4.2 \pm 1.2$ & $3.0 \pm 0.6$ & $3.5 \pm 0.7$ & $3.3 \pm 0.8$ \\
\hline$\# 2$ & $3.1 \pm 0.2$ & $2.9 \pm 0.3$ & $2.9 \pm 0.2$ & $2.5 \pm 0.2$ & $3.4 \pm 0.3$ & $3.6 \pm 0.5$ \\
\hline$\# 3$ & $4.1 \pm 0.6$ & $3.8 \pm 0.5$ & $3.6 \pm 0.5$ & $3.8 \pm 0.6$ & $3.5 \pm 0.2$ & $3.3 \pm 0.5$ \\
\hline$\# 4$ & $3.8 \pm 0.7$ & $4.0 \pm 0.9$ & $3.8 \pm 0.7$ & $3.5 \pm 0.7$ & $2.7 \pm 0.2$ & $2.6 \pm 0.3$ \\
\hline$\# 5$ & $3.7 \pm 0.6$ & $3.4 \pm 0.5$ & $3.2 \pm 0.6$ & $2.8 \pm 0.3$ & $2.6 \pm 0.3$ & $2.5 \pm 0.3$ \\
\hline$\# 7$ & $3.7 \pm 0.6$ & $3.2 \pm 0.5$ & $2.8 \pm 0.5$ & $2.9 \pm 0.5$ & $2.6 \pm 0.4$ & $2.4 \pm 0.3$ \\
\hline$\# 8$ & $3.7 \pm 1.4$ & $3.6 \pm 1.1$ & $3.4 \pm 1.3$ & $3.7 \pm 0.5$ & $3.3 \pm 0.3$ & $3.4 \pm 0.4$ \\
\hline
\end{tabular}

Values are given in $\mathrm{m} \mathrm{s}^{-1}$ (mean $\pm \mathrm{SD}$ for 10 cardiac cycles) for three repetitive measurements (1 to 3 ) per subject and 2 positions (i.e., one section covering the ascending and descending aorta). 
Table 2. Mean aortic pulse wave velocities using real-time and cine phase-contrast MRI.

\begin{tabular}{|c|c|c|c|c|}
\hline Acquisition & Analysis & 2 Positions & 3 Positions & 4 Positions \\
\hline \hline \multirow{3}{*}{ Real-time } & \multirow{3}{*}{ TTU } & $3.9 \pm 0.6$ & $3.9 \pm 0.4$ & $3.8 \pm 0.4$ \\
\cline { 2 - 5 } & & $3.6 \pm 0.5$ & & \\
& \multirow{3}{*}{ XCor } & $3.5 \pm 0.5$ & $3.3 \pm 0.4$ & $3.2 \pm 0.4$ \\
& & $3.0 \pm 0.5$ & & $3.8 \pm 0.6$ \\
\hline \multirow{2}{*}{ Cine } & TTU & $2.9 \pm 0.5$ & $3.5 \pm 0.5$ & $3.7 \pm 0.5$ \\
\cline { 2 - 5 } & XCor & $3.3 \pm 0.5$ & $3.6 \pm 0.6$ \\
\hline
\end{tabular}

Values are given in $\mathrm{m} \mathrm{s}^{-1}$ (mean $\pm \mathrm{SD}$ ) for 8 subjects and 10 cardiac cycles (real-time data). The real-time data for 2 positions represent 3 repetitions (compare Table $\mathbf{1}$ ).

between repetitive measurements as well as breathinginduced changes between inspiration and expiration. This underlying sensitivity of real-time PC MRI to respiration is demonstrated in Fig. (3) for a single subject (single section) and 10 consecutive cardiac cycles. The traces represent mean velocities in the ascending and descending aorta together with the resulting PWV values as a function of time for a period covering about two respiratory cycles. Quantitative differences (i.e., maximum to minimum PWV values) are on the order of $1 \mathrm{~m} \mathrm{~s}^{-1}$.

\section{DISCUSSION}

As shown in Table $\mathbf{1}$ individual real-time PWV values for our group of healthy young volunteers are highly reproducible on an individual basis and regardless of the analysis method. Beat-to-beat variations suggest a pronounced sensitivity to physiological alterations. The resulting aortic PWW values compare well with literature data which nevertheless are rather diverse and cover a broad range, also because PWV values tend to increase linearly with age even in healthy subjects $[1,2]$. For example, while early cine PC MRI studies [15, 33, 39] mainly used the time-to-foot method abandoned here, a more recent cine PC MRI study [42] introduced TTU and XCor analyses and reported 5.1 to 5.3 $\mathrm{m} \mathrm{s}^{-1}$ for 2D PC MRI as well as 3.8 to $4.8 \mathrm{~m} \mathrm{~s}^{-1}$ for 4D PC MRI and middle-aged subjects.

Here, consistent PWV values in the range from 3.5 to 3.9 $\mathrm{m} \mathrm{s}^{-1}$ were obtained for both real-time and cine PC MRI in conjunction with a TTU analysis. For the XCor method, the PWV values for real-time PC MRI are up to $10 \%$ lower than for cine PC MRI. However, in the absence of a true gold standard, it seems impossible to decide about the "best" or most reliable analysis technique. Moreover, this discussion is outside the scope of the present study which emphasizes real-time vs cine PC MRI.

Previous PWV work [15] suggested the measurement of flow velocities from multiple locations along the aorta for a more accurate analysis which, for example, minimizes the putative influence of turbulent blood flow close to the aortic arch. Most remarkably, however, for any chosen acquisition and analysis technique the PWV values obtained in the present study for 2, 3 or 4 positions were very consistent and comparable. This also includes a very reproducible determination of mean PWV values from real-time PC MRI measurements of only a single slice covering the ascending and descending aorta. While such a strategy might also be preferable because the data for the two analyzed positions stem from the same cardiac cycles, its successfulness depends on the shape of the aorta and the ability to place a section for measuring through-plane flow in both locations within the acceptable limits of 10 to $15^{\circ}$ angulation [43].

A limitation of the present and previous 2D PC MRI studies is the manual determination of aortic distances from a single sagittal plane. Although simple and performed with consensus, the approach may be prone to errors because of the complex anatomy of the aorta and variations during breathing. However, when analyzing the distance for specific breathing conditions (i.e., during breathhold after inhalation or exhalation), the differences between two locations in the ascending and descending aorta (same section) turned out to be surprisingly small (about 3\%, data not shown) and unable to account for the PWV modulations during free breathing. The latter effect has to be ascribed to the lowered intrathoracic pressure during inspiration which causes increased flow velocities in the ascending aorta [35].

\section{CONCLUSION}

Aortic PWV values may be obtained for single cardiac cycles by real-time PC MRI with sufficiently high spatial and temporal resolution. Respective analyses were performed for two different analysis methods and up to 4 locations along the aorta. While mean PWV values averaged across multiple cardiac cycles were found to be reproducible and consistent for 2, 3 and 4 locations, the beat-to-beat variability of PWV values from individual cardiac cycles revealed a remarkable sensitivity to breathing with highest values during inspiration, i.e. lowest intrathoracic pressure. In future, real-time PC MRI determinations of PWV are expected to markedly reduce examination times. Moreover, 


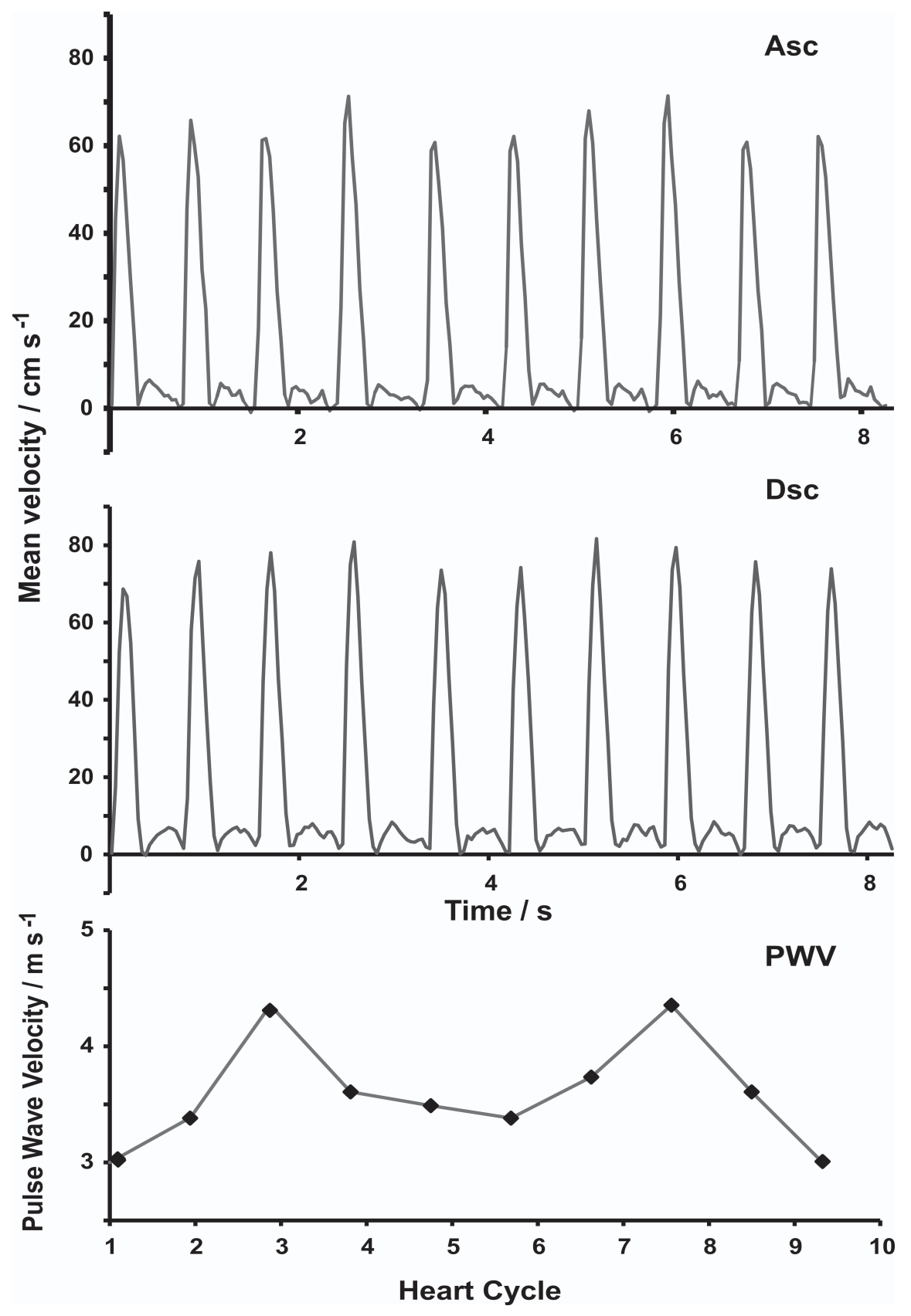

Fig. (3). Mean blood flow velocities obtained from real-time phase-contrast MRI (single subject, single section) in the ascending (Asc) and descending aorta (Dsc) as well as resulting pulse wave velocities (PWV) as a function of time for 10 cardiac cycles.

real-time PC MRI will allow for studying PWV variations in response to breathing maneuvers $[27,35,36]$ or protocols of physical exercise which may aid in the diagnosis of patients with suspected aortic stiffness.

\section{CONFLICT OF INTEREST}

JF holds a patent on the NLINV reconstruction method.

\section{ACKNOWLEDGEMENTS}

We are grateful for financial support (A.A.J.) by the DZHK (German Centre for Cardiovascular Research) and BMBF (German Ministry of Education and Research).

\section{REFERENCES}

[1] Schlatmann TJ, Becker AE. Histologic changes in the normal aging aorta: implications for dissecting aortic aneurysm. Am J Cardiol 1977; 39: 13-20.

[2] Mohiaddin RH, Firmin DN, Longmore DB. Age-related changes of human aortic flow wave velocity measured noninvasively by magnetic resonance imaging. J Appl Physiol 1993; 74: 492-7.

[3] Hansen TW, Staessen JA, Torp-Pedersen C, et al. Prognostic value of aortic pulse wave velocity as index of arterial stiffness in the general population. Circulation 2006; 113: 664-70.

[4] Mohiaddin RH, Longmore DB. MRI studies of atherosclerotic vascular disease: structural evaluation and physiological measurements: Br Med Bull 1989; 45: 968-90.

[5] Laurent S, Katsahian S, Fassot C, et al. Aortic stiffness is an independent predictor of fatal stroke in essential hypertension. Stroke 2004; 34: 1203-6. 
[6] Laurent S, Boutouyrie P, Asmar R, et al. Aortic stiffness is an independent predictor of all-cause and cardiovascular mortality in hypertensive patients. Hypertension 2001; 37: 1236-41.

[7] Latham RD, Westerhof N, Sipkema P, Rubal BJ, Reuderink P, Murgo JP. Regional wave travel and reflections along the human aorta - a study with six simultaneous micromanometric pressures. Circulation 1985; 72: 1257-69.

[8] Chen $\mathrm{CH}$, Nevo E, Fetics B, et al. Estimation of central aortic pressure waveform by mathematical transformation of radial tonometry pressure: validation of generalized transfer function. Circulation 1997; 95: 1827-36.

[9] Wilkinson IB, Fuchs SA, Jansen IM, Murray GD, Cockcroft JR, Webb DJ. The reproducibility of pulse wave velocity and augmentation index measured by pulse wave analysis. J Hypertens 1998; 16: 2079-84.

[10] Katakami N, Osonoi T, Takahara M, et al. Clinical utility of brachial-ankle pulse wave velocity in the prediction of cardiovascular events in diabetic patients. Cardiovasc Diabetol 2014; 13: 128.

[11] Pelc NJ, Herfkens RJ, Shimakawa A, Enzmann DR. Phase contrast cine magnetic resonance imaging. Magn Reson Q 1991; 7: 229-54.

[12] Gatehouse PD, Keegan J, Crowe LA, et al. Applications of phasecontrast flow and velocity mapping in cardiovascular MRI. Eur Radiol 2005; 15: 2172-84.

[13] Pelc NJ, Sommer FG, Li KC, Brosnan TJ, Herfkens RJ, Enzmann DR. Quantitative magnetic resonance flow imaging. Magn Reson Q 1994; 32: 330-4.

[14] Markl M, Chan FP, Alley MT, et al. Time-resolved threedimensional phase-contrast MRI. J Magn Reson Imaging 2003; 17: 499-506.

[15] Markl M, Wallis W, Brendecke S, Simon J, Frydrychowicz A, Harloff A. Estimation of global aortic pulse wave velocity by flowsensitive 4D MRI. Magn Reson Med 2010; 63: 1575-82.

[16] Eichenberger AC, Schwitter J, McKinnon GC, Debatin JF, von Schulthess GK. Phase-contrast echo-planar MR imaging - realtime quantification of flow and velocity patterns in the thoracic vessels induced by Valsalva's maneuver. J Magn Reson Imaging 1995; 5: 648-55.

[17] Gatehouse PD, Firmin DN, Collins S, Longmore DB. Realtime blood flow imaging by spiral scan phase velocity mapping. Magn Reson Med 1994; 31(5): 504-12.

[18] Nayak KS, Pauly JM, Kerr AB, Hu BS, Nishimura DG. Real-time color flow MRI. Magn Reson Med 2000; 43: 251-8.

[19] Nezafat R, Kellman P, Derbyshire JA, Mcveigh E. Real-time blood flow imaging using autocalibrated spiral sensitivity encoding. Magn Reson Med 2005; 54: 1557-61.

[20] Steeden JA, Atkinson D, Taylor AM, Muthurangu V. Assessing vascular response to exercise using a combination of real-time spiral phase contrast MR and noninvasive blood pressure measurements. J Magn Reson Imaging 2010; 31: 997-1003.

[21] Thompson RB, Elliot RM. Flow-gated phase-contrast MRI using radial acquisitions. Magn Reson Med 2004; 52: 598-604.

[22] Johnson KM, Lum DP, Turski PA, Block WF, Mistretta CA, Wieben O. Improved 3D phase contrast MRI with off-resonance corrected dual echo VIPR. Magn Reson Med 2008; 60: 1329-36.

[23] Shankaranarayanan A, Simonetti OP, Laub G, Lewin JS, Duerk JL. Segmented k-space and real-time cardiac cine MR imaging with radial trajectories. Radiology 2001; 221: 827-36.

[24] Pruessmann KP, Weiger M, Scheidegger MB, Boesiger P. SENSE: sensitivity encoding for fast MRI. Magn Reson Med 1999; 42: 95262.

[25] Griswold MA, Jakob PM, Heidemann RM, et al. Generalized autocalibrating partially parallel acquisitions (GRAPPA). Magn Reson Med 2002; 47: 1202-10.
[26] Bock M, Schad LR, Mueller E, Lorenz WJ. Pulsewave velocity measurement using a new real-time MR method. Magn Reson Imaging 1995; 13: 21-9.

[27] Gaddum NR, Schaeffter T, Buehrer M, et al. Beat to beat variation in pulse wave velocity during breathing maneuvers. Magn Reson Med 2014; 72: 202-10.

[28] Uecker M, Hohage T, Block KT, Frahm J. Image reconstruction by regularized nonlinear inversion - joint estimation of coil sensitivities and image content. Magn Reson Med 2008; 60: 674-82.

[29] Uecker M, Zhang S, Voit D, Karaus A, Merboldt KD, Frahm J. Real-time MRI at a resolution of $20 \mathrm{~ms}$. NMR Biomed 2010; 23: 986-94.

[30] Zhang S, Uecker M, Voit D, Merboldt KD, Frahm J. Real-time cardiovascular magnetic resonance at high temporal resolution radial FLASH with nonlinear inverse reconstruction. J Cardiovasc Magn Reson 2010; 12: 39-46.

[31] Voit D, Zhang S, Unterberg-Buchwald C, Sohns JM, Lotz J, Frahm $\mathrm{J}$. Real-time cardiovascular magnetic resonance at $1.5 \mathrm{~T}$ using balanced SSFP and $40 \mathrm{~ms}$ resolution. J Cardiovasc Magn Reson 2013; 15: 79.

[32] Zhang S, Joseph AA, Voit D, et al. Real-time MRI of cardiac function and flow - recent progress. Quant Imaging Med Surg 2014; 4: 313-29.

[33] Joseph AA, Merboldt KD, Voit D, et al. Real-time phase-contrast MRI of cardiovascular blood flow using undersampled radial fast low-angle shot and nonlinear inverse reconstruction. NMR Biomed 2012; 25: 917-24.

[34] Joseph AA, Kowallick JT, Merboldt KD, et al. Real-time flow MRI of the aorta at a resolution of $40 \mathrm{~ms}$. J Magn Reson Imaging 2014 40: 206-13

[35] Fasshauer M, Joseph AA, Kowallick JT, et al. Real-time phasecontrast flow MRI of hemodynamic changes in the ascending aorta and superior vena cava during Mueller maneuver. Clin Radiol 2014; 69: 1066-71.

[36] Kowallick JT, Joseph AA, Unterberg-Buchwald C, et al. Real-time phase-contrast flow MRI of the ascending aorta and superior vena cava as a function of intrathoracic pressure (Valsalva manoeuvre). Br J Radiol 2014; 87(1042): 10.1259/bjr.20140401.

[37] Frahm J, Schaetz S, Untenberger M, et al. On the temporal fidelity of nonlinear inverse reconstructions for real-time MRI - The motion challenge. Open Med Imaging J 2014; 8: 1-7.

[38] Schaetz S, Uecker M. A multi-GPU programming library for realtime applications. In: Xiang Y, Stojmenovic I, Apduhan B, Wang G, Nakano K, Zomaya A, Eds. Algorithms and Architectures for Parallel Processing; Lecture Notes in Computer Science. Springer: Berlin Heidelberg 2012; 7439: pp. 114-128.

[39] Vulliemoz S, Stergiopulos N, Meuli R. Estimation of local aortic elastic properties with MRI. Magn Reson Med 2002; 47: 649-54.

[40] Fielden SW, Fornwalt BK, Jerosch-Herold M, Eisner RL, Stillman AE, Oshinski JN. A new method for the determination of aortic pulse wave velocity using cross-correlation on 2D PCMR velocity data. J Magn Reson Imaging 2008; 27: 1382-7.

[41] Grotenhuis HB, Westenberg JJM, Steendijk P, et al. Validation and reproducibility of aortic pulse wave velocity as assessed with velocity-encoded MRI. J Magn Reson Imaging 2009; 30: 521-6.

[42] Wentland AL, Wieben O, Francois CJ, et al. Aortic pulse wave velocity measurements with undersampled $4 \mathrm{D}$ flow-sensitive MRI - comparison with 2D and algorithm determination. J Magn Reson Imaging 2013; 37: 853-9.

[43] Lotz J, Meier C, Leppert A, Galanski M. Cardiovascular flow measurement with phase-contrast MR imaging - Basic facts and implementation. Radiographics 2002, 22: 651-71.

(C) Joseph et al.; Licensee Bentham Open.

This is an open access article licensed under the terms of the Creative Commons Attribution Non-Commercial License (http://creativecommons.org/licenses/by-nc/3.0/) which permits unrestricted, non-commercial use, distribution and reproduction in any medium, provided the work is properly cited. 